\title{
EVALUACIÓN Y SELECCIÓN DE UN INHIBIDOR MULTISCALE PARA PREVENIR LA FORMACIÓN DE INCRUSTACIONES INORGÁNICAS EN UN CAMPO PETROLERO COLOMBIANO
}

\author{
Carolina León Vanegas ${ }^{1 *}$, Hernando Buendia ${ }^{2}$, Luis Felipe Carrillo ${ }^{3}$ \\ *A quien debe dirigirse la correspondencia
}

\section{RESUMEN}

El Daño de Formación por depositación de escamas inorgánicas constituye el de mayor frecuencia y de mayor incidencia sobre la producción en Campos Colombianos, ocasiona considerables pérdidas económicas debido a las restricciones en el flujo, trabajos de reacondicionamiento de pozos y a la producción diferida. Por lo tanto, se hace necesario la implementación de técnicas de inhibición que eviten su formación y posterior depositación.

El presente trabajo consistió en evaluar a nivel de laboratorio tres inhibidores disponibles comercialmente en Colombia (Inhibidor I, Inhibidor II e Inhibidor III) y seleccionar el de mejor comportamiento para su aplicación en un tratamiento por squeeze en fondo de pozo en el Campo Cupiagua.

Para la selección del inhibidor se realizaron pruebas experimentales de interacción fluido-fluido: pruebas de Sludge, mojabilidad visual, rompimiento de emulsiones y compatibilidad. También se realizaron pruebas técnicas para determinar la estabilidad térmica y la concentración mínima de inhibidor a condiciones dinámicas y de yacimiento. Finalmente se determinó la compatibilidad entre el inhibidor seleccionado con los fluidos y la roca de la formación a través de pruebas de interacción fluido-roca. Estas pruebas permitieron establecer que el inhibidor I se clasifica como apto para inyección por squeeze en el pozo CUP XL4 del Campo Cupiagua, el cual presenta evidencias de problemas severos de disminución de producción por depositación de incrustaciones de carbonato de calcio y sulfato de bario.

Palabras clave: Daño de Formación, Inhibidor de Incrustaciones, Normas Técnicas Internacionales

\section{EVALUATION AND SELECTION OF A MULTISCALE INHIBITOR TO PREVENT INORGANIC SCALING IN A COLOMBIAN OIL FIELD.}

\begin{abstract}
Formation damage produced by inorganic scaling is the most frequently and has the major impact on the Colombian oil fields. It causes important economic losses due to flow restrictions, workover operations and production losses. Therefore it is necessary the implementation of inhibition techniques to prevent scaling.

This job evaluated in the laboratory three commercially available inhibitors in Colombia (Inhibitor I, Inhibitor II and Inhibitor III) and selected the best behavior of them, for its application in a bottom hole squeeze treatment at the Cupiagua Field.
\end{abstract}

1. Grupo de Investigación: Modelamiento de Procesos de Hidrocarburos, GMPH. Universidad Industrial de Santander, UIS, Carrera 27 Calle 9 Bucaramanga, Colombia. karitoleon@gmail.com.

2. Escuela de Ingeniería de Petróleos, Universidad Industrial de Santander, UIS, Carrera 27 calle 9, Bucaramanga, Colombia.

3. Escuela de Ingeniería de Petróleos, Universidad Industrial de Santander, UIS, Carrera 27 calle 9, Bucaramanga, Colombia. 
Fluid-Fluid interaction experimental tests were performed to select the inhibitor: Sludge, Visual Wettability, Emulsions and Compatibility were also performed to determinate the thermal stability and the minimum inhibitor concentration at dynamic and reservoir conditions. Finally, Compatibility was determinated among the selected inhibitor and the formation rock and fluids through rock-fluid interaction tests. These tests stablished the inhibitor I as the best one and the optimum for the squeeze injection at CUP XL4 well, of Cupiagua Field, which presents several problems of production decline because calcium carbonate and barium sulfate scaling.

Keywords: Formation Damage, Scale Inhibitor, International Technical Standards

\section{INTR UCCIÓN}

La formación de incrustaciones inorgánicas constituye un grave problema para la industria del petróleo y gas durante la producción de los fluidos del pozo. Las incrustaciones pueden depositarse sobre cualquier superficie, de manera que una vez que se forman los primeros cristales, estos continúan creciendo, formando una capa cada vez más gruesa a menos que sean tratados. Pueden ocasionar un bloqueo en la región cercana al pozo de perforación, o en el mismo pozo, causando daño a la formación y pérdida de productividad del pozo. También se pueden depositar sobre los equipos de superficie causando su mal funcionamiento o pueden aparecer en cualquier parte a lo largo de los tubos de producción, reduciendo el diámetro interno y bloqueando el flujo, e incluso pueden formarse en las plantas de tratamiento. ${ }^{[1]}$

Las consecuencias de los depósitos de incrustaciones son: disminución de la producción, problemas con la inyección de agua, restricción en el flujo, trabajos de reacondicionamiento de pozos debido a reducción en la producción, reparación y mantenimiento de los equipos de superficie, y consumo de aditivos químicos para la limpieza de los equipos incrustados. ${ }^{[2]}$

Los efectos que provocan las incrustaciones pueden resultar dramáticos e inmediatos. Cualquiera que sea la forma de precipitación, el flujo se reduce a tal punto que puede producirse el abandono del pozo. Por tanto, los costos por la formación de incrustaciones son muy significativos, y la solución a este tipo de problemas le cuesta a la industria cientos de millones de dólares por año en términos de mantenimiento y pérdidas de producción cuando se deja de extraer crudo. ${ }^{[3]}$.

El costo directo de remover las incrustaciones de un pozo puede alcanzar los 250.000 dólares, más el costo de la producción diferida, que resulta aún más elevado. Por todo lo anterior, es mejor prevenir la formación de incrustaciones, manteniendo los pozos productores en buen estado, lo cual constituye la forma más eficiente de producir hidrocarburos. ${ }^{[4][5]}$
En este proyecto de investigación se describe una metodología para la evaluación de inhibidores de incrustación, la cual permite seleccionar el mejor inhibidor para ser inyectado en la formación de interés. Para este propósito se inició con una caracterización básica de los inhibidores como criterio de control de calidad. Seguidamente se realizaron diferentes pruebas con el fin de establecer la compatibilidad de los inhibidores con crudo, arena y agua de la formación. Posteriormente se determinó la eficiencia de los inhibidores mediante la realización de pruebas técnicas que simulaban las condiciones del yacimiento. Y finalmente se seleccionó el mejor inhibidor, de acuerdo a los resultados de las pruebas anteriores para determinar la compatibilidad de éste con la roca de la formación mediante desplazamiento dinámico fluido-roca.

\section{METODOLOGÍA}

Caracterización_Básica. La etapa de caracterización básica contempla las siguientes pruebas: densidad, $\mathrm{pH}$, solubilidad, índice de refracción, viscosidad y espectro infrarrojo. Estas pruebas se realizaron con el fin de establecer un control de calidad a los inhibidores recibidos.

Eficiencia Estática. Esta prueba se rige bajo la norma NACE TM0374-90. Consiste en determinar un intervalo de concentración de inhibidor necesario, para evitar la formación de cristales de sulfato de bario y/o carbonato de calcio, a presión atmosférica y $95^{\circ} \mathrm{C}$. Para su realización se prepararon dos salmueras, una compuesta por cationes (S-1) y otra por aniones (S2), que al mezclarse en proporción 1:1 reproduce una salmuera equivalente a la de interés.

Se dosificaron en frascos de cierre hermético 5, 10, 15, 20 y $50 \mathrm{ppm}$ de cada producto, en $50 \mathrm{ml}$ de la salmuera $\mathrm{S}-2$; en frascos aparte, se midieron $50 \mathrm{ml}$ de la salmuera S-1 y finalmente se colocaron todas las soluciones en un horno a $95^{\circ} \mathrm{C}$. Al cabo de una hora se vertió el contenido de los frascos con S-1 sobre S-2 y se mantuvieron a ésta temperatura por 24 horas. Se realizaron observaciones 
detalladas de la cantidad y apariencia del precipitado formado a los tiempos de 1, 2, 6 y 24 horas.

Interacción Fluido-Fluido. Esta etapa contempla las siguientes pruebas: sludge, mojabilidad visual, rompimiento de emulsiones, compatibilidad con y sin envenenamiento. Las pruebas que forman parte de esta etapa se rigen bajo la Norma API RP-42 de 1990. Se realizaron con el propósito de evaluar la compatibilidad entre los inhibidores y los fluidos del pozo Cupiagua XL4.

Sludge. Esta prueba se realizó para evaluar la tendencia que tienen algunos crudos en contacto con sustancias, generalmente ácidas, a precipitar sólidos llamados Sludge. El procedimiento consistió en adicionar el inhibidor en proporción 1:1 con aceite crudo libre de sólidos y emulsión, agitar vigorosamente durante 60 segundos y someter a temperatura de $95{ }^{\circ} \mathrm{C}$ durante 24 horas. $\mathrm{Al}$ término de las 24 horas, se pasó la mezcla a través de una malla de 100 mesh y se examinó si quedaron sólidos remanentes en ella. Se lavó la malla alternadamente con agua tibia y aceite mineral para remover emulsiones y parafinas sin remover los Sludge que pudieran ser causados por el Inhibidor.

Mojabilidad Visual. Esta prueba se realizó para determinar cualitativamente la tendencia humectante del Inhibidor. En $50 \mathrm{ml}$ de Inhibidor puro se adicionaron 10 cc de arena Ottawa limpia, de tamaño de partícula 40 a 60 mesh, después de media hora en contacto se decantó la solución y se separó la arena.

En probetas diferentes se colocó agua de Villatina y aceite de referencia. Se adicionó la arena dentro de cada una de las probetas, observando la dispersión relativa de las partículas o su tendencia a formar grumos en la fase acuosa y en la fase aceite.

Rompimiento de Emulsiones. Para la realización de esta prueba se dosificó el Inhibidor al $20 \%$ en la salmuera sintética de composición eléctrica equivalente al agua de formación del pozo Cupiagua XL4, diluida al 50\% con agua destilada; se preparó $100 \mathrm{ml}$ de mezcla crudo Cupiagua XL4 - inhibidor en tres proporciones: 25:75, 50:50 y $75: 25$. A cada mezcla se adicionaron 2.5 gramos de finos sintéticos preparados (se utilizó una mezcla de $85 \%$ de arena Ottawa y $15 \%$ de Bentonita para remplazar los finos de la formación Barco y Mirador, 200 mesh). Posteriormente y se agitó a $14000 \mathrm{rpm}$, durante 30 segundos, y se realizó observaciones a los 15 minutos, 1 y
24 horas registrando el volumen de agua de rompimiento y el aspecto de la solución acuosa de la emulsión.

Compatibilidad. La prueba de compatibilidad consta de dos etapas, la primera evalúa la compatibilidad con la salmuera y la segunda la compatibilidad con el crudo.

Compatibilidad con Salmuera. Para el desarrollo de esta prueba se preparó una salmuera sintética, de composición eléctrica equivalente al agua de formación del pozo Cupiagua XL4 y se diluyó al 50\% con agua destilada. En frascos de cierre hermético se dosificaron cada uno de los inhibidores, al 1\%, 2,5\%, $5 \%, 10 \%$ y $20 \%$, en la salmuera sintética preparada; se agitaron a $14.000 \mathrm{rpm}$ por 60 segundos y se calentaron gradualmente, empezando con $50^{\circ} \mathrm{C}$ seguido por $60^{\circ} \mathrm{C}, 80^{\circ} \mathrm{C}$, y $95^{\circ} \mathrm{C}$, todos en intervalos de 1 hora. La temperatura final $\left(95^{\circ} \mathrm{C}\right)$ se mantuvo hasta completar 24 horas de evaluación. Para cada Temperatura se realizaron observaciones, detallando el aspecto de la solución y la cantidad de precipitados.

Compatibilidad con Crudo. En frascos de cierre hermético se realizaron mezclas 1:1 entre el crudo del pozo Cupiagua XL4 y las soluciones que no presentaron incompatibilidad en la prueba con salmuera; se agitaron a $14.000 \mathrm{rpm}$ por 60 segundos y se colocaron en un horno a $95^{\circ} \mathrm{C}$. Igual que en la etapa anterior, se realizaron observaciones a los tiempos de 1,2 y 24 horas de evaluación, detallando la detergencia, la calidad en la separación de las fases y los signos de incompatibilidad.

Compatibilidad Envenenando con Calcio, Hierro y Bario. Esta prueba evalúa la compatibilidad del inhibidor con los fluidos de formación del pozo Cupiagua XL4, en presencia de 5.000 ppm de Calcio, $5.000 \mathrm{ppm}$ de Hierro y $500 \mathrm{ppm}$ de Bario. Consta de dos etapas: compatibilidad con la salmuera envenenada y compatibilidad con el crudo CUP XL-4.

Compatibilidad con Salmuera envenenada. Para el desarrollo de esta prueba se siguió el mismo procedimiento descrito anteriormente en la prueba de compatibilidad, modificando únicamente la dosificación del inhibidor, que para este caso fue de 20 $\%$. Adicionalmente se envenenó la salmuera preparada con 5.000 ppm de calcio utilizando $\mathrm{CaCl}_{2} \cdot 2 \mathrm{H}_{2} \mathrm{O}$.

Compatibilidad con Crudo. Para el desarrollo de esta prueba se siguió el mismo procedimiento descrito anteriormente en la prueba de compatibilidad. Para las 
pruebas envenenando con Hierro y Bario se siguió el mismo procedimiento utilizado para el Calcio y se usaron los reactivos $\mathrm{FeCl}_{2} 4 \mathrm{H}_{2} \mathrm{O}$ y $\mathrm{BaCl}_{2} 2 \mathrm{H}_{2} \mathrm{O}$ respectivamente.

\section{Pruebas TÉCNICAS.}

Estabilidad Térmica. Para ésta evaluación se prepararon soluciones de los inhibidores al 5\% como producto recibido en la salmuera sintética Cupiagua XL4 y se colocaron en reactores para digestión (bombas PAAR), inertes y herméticos, a una temperatura de 122 ${ }^{\circ} \mathrm{C}$ y una presión de 4.500 psi durante 5 días. Al final del período los reactores se dejaron enfriar y se tomaron los espectros IR de cada muestra. Por comparación del espectro inicial con el final, se determinó si el producto sufrió, o no, cambios en su composición.

Eficiencia Dinámica. Para determinar la concentración mínima de inhibidor se utilizó un equipo Coil Tubing System (Figura 1), el cual se basa en el método de bloqueo de tubo. Este equipo es capaz de medir pequeños cambios en la presión debido a la acumulación de incrustaciones en un capilar en forma de espiral (capillary coil).

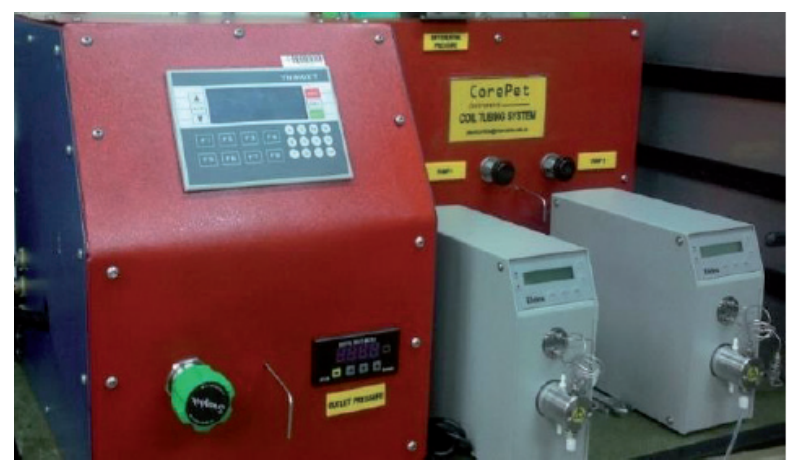

Figura 1. Equipo Coil Tubing System.

La metodología general consistió en bombear dos salmueras incompatibles a través de un capilar en espiral que se mantuvo a las condiciones de temperatura y presión en las que probablemente se encuentra en el sistema de producción. La acumulación de incrustaciones en el capilar genera un diferencial de presión a través de este, el cual es usado como una medida de la depositación de las incrustaciones al interior de la tubería. Una vez que se inyectó el inhibidor, su efecto fue monitoreado mediante este parámetro. Para obtener la MIC, la concentración del inhibidor de incrustaciones se redujo continuamente hasta que el diferencial de presión empezó a aumentar. En ese momento se incrementó la concentración del inhibidor para establecer el valor al cual no se producía la depositación de incrustaciones. El menor valor de concentración que era capaz de inhibir la formación de incrustaciones en la tubería de espiral (coil tubing), se denominó concentración mínima de inhibidor (MIC). En la Tabla 1 se registra la concentración de las salmueras catiónica y aniónica para la prueba de eficiencia dinámica.

Tabla 1. Concentración de las salmueras para la prueba de Eficiencia Dinámica en el equipo Coil Tubing System.

AGUA DE FORMACIÓN

\begin{tabular}{cccc}
\hline IONES & CONCENTRACIÓN $(\mathbf{m g} / \mathbf{L})$ & $\begin{array}{c}\text { SALES } \\
\text { ADICIONADAS }\end{array}$ & $\begin{array}{c}\text { CONCENTRACIÓN } \\
\text { DE LAS SALES PARA LA PRUEBA (g/L) }\end{array}$ \\
\hline $\mathbf{N a}^{+}$ & 3908 & $\mathrm{NaCl}$ & 19.87 \\
$\mathbf{K}^{+}$ & 162 & $\mathrm{KCl}$ & 0.62 \\
$\mathbf{C a}^{+2}$ & 687 & $\mathrm{CaCl}_{2} \cdot 2 \mathrm{H}_{2} \mathrm{O}$ & 5.04 \\
$\mathbf{M g}^{+2}$ & 198 & $\mathrm{MgCl}_{2} \cdot 6 \mathrm{H}_{2} \mathrm{O}$ & 3.31 \\
\hline $\mathbf{F e}^{+3}$ & 0.1 & $\mathrm{FeCl}_{3}$ & 0.005812 \\
$\mathbf{B a}^{+2}$ & 20 & $\mathrm{BaCl}_{2} \cdot 2 \mathrm{H}_{2} \mathrm{O}$ & 0.07 \\
\hline $\mathbf{S r}^{+2}$ & 52 & - & - \\
\hline$\left(\mathbf{H C O}_{3}\right)^{-\mathbf{1}}$ & 664 & $\mathrm{NaHCO}_{3}$ & 1.83 \\
\hline $\left.\mathbf{S O}_{4}\right)^{-2}$ & 29 & $\mathrm{Na}_{2} \mathrm{SO}_{4}$ & 0.08576 \\
\hline $\mathbf{C l}^{-1}$ & 8589 & & \\
\hline
\end{tabular}




\section{INTERACCIÓN FLUIDO - ROCA}

Restauración de Mojabilidad. Antes de la prueba de desplazamiento se realizó una Restauración de la Mojabilidad al Plug, con el fin de recuperar su tendencia natural a dejarse humectar por un fluido, cuando se encuentra en presencia de varios fluidos inmiscibles.

Desplazamiento. Esta prueba se realizó con el fin de determinar la compatibilidad entre el inhibidor, los fluidos y la roca de la formación. Para esta prueba se tuvo en cuenta las condiciones de presión y temperatura de yacimiento. El plug se montó en un equipo Liquid Fluid System Automatizado, se suministraron condiciones de presión de confinamiento (4000 psi), temperatura de yacimiento $\left(120^{\circ} \mathrm{C}\right)$ y presión de inyección (alrededor de 500 psi). La prueba consistió en inyectar en el plug los sistemas de fluidos por baches, cuyas características y volúmenes fueron establecidas previamente mediante un protocolo de desplazamiento (ver Tabla 2).

Tabla 2. Protocolo de desplazamiento para el Inhibidor seleccionado.

\begin{tabular}{|c|c|c|}
\hline FLUIDO A DESPLAZAR & SENTIDO & $\begin{array}{c}\text { VOLUMEN } \\
\text { POROSO } \\
\text { Vp }\left(\mathrm{cm}^{3}\right)\end{array}$ \\
\hline Crudo & Producción & Estabilidad \\
\hline Salmuera & Producción & Estabilidad \\
\hline Crudo & Producción & Estabilidad \\
\hline Preflujo Orgánico 1 & Inyección & 1 \\
\hline Preflujo Orgánico 2 & Inyección & 1 \\
\hline Preflujo Orgánico 3 & Inyección & 1 \\
\hline \multicolumn{3}{|c|}{ Remojo 4 horas } \\
\hline Inhibidor multiscale & Inyección & 5 \\
\hline \multicolumn{3}{|c|}{ Remojo 12 horas } \\
\hline Salmuera & Producción & 250 \\
\hline Preflujo Orgánico 3 & Producción & 1 \\
\hline Preflujo Orgánico 2 & Producción & 1 \\
\hline Preflujo Orgánico 1 & Producción & 1 \\
\hline Crudo & Producción & Estabilidad \\
\hline
\end{tabular}

La dosificación de inhibidor para esta prueba fue establecida por la compañía fabricante del producto a una concentración de 7,5\% en salmuera de $\mathrm{KCl}$ al $3 \%$.

\section{RESULTADOS}

Eficiencia Estática. Los resultados de la prueba de eficiencia estática se resumen en la Tabla 3. La interpretación de los resultados está basada en la siguiente nomenclatura.
No presentó precipitado al término de 24 horas.

Presentó precipitado al término de 24 horas

Tabla 3. Resultados de la prueba de eficiencia estática.

\begin{tabular}{|c|c|c|c|c|c|}
\hline \multirow{2}{*}{ PRODUCTO } & \multicolumn{5}{|c|}{ CONCENTRACIÓN } \\
\cline { 2 - 6 } & $\mathbf{5}$ & $\mathbf{1 0}$ & $\mathbf{1 5}$ & $\mathbf{2 0}$ & $\mathbf{5 0}$ \\
\hline Inhibidor I & $\bigcirc$ & $\bigcirc$ & $\bigcirc$ & $\bigcirc$ & $\bigcirc$ \\
\hline Inhibidor III & $\bigcirc$ & $\bigcirc$ & $\bigcirc$ & $\bigcirc$ & $\bigcirc$ \\
\hline Inhibidor II & $\bigcirc$ & $\bigcirc$ & $\bigcirc$ & $\bigcirc$ & $\bigcirc$ \\
\hline
\end{tabular}

De acuerdo con los resultados mostrados en la Tabla 3, el inhibidor I tuvo el mejor comportamiento. La mínima concentración de Inhibidor I necesaria para evitar la formación de precipitado a $95^{\circ} \mathrm{C}$ y presión atmosférica fue de $5 \mathrm{ppm}$; para el inhibidor III la concentración mínima esta en un rango (mayor de $20 \mathrm{ppm}$ y menor o igual a $50 \mathrm{ppm}$ ), y para el inhibidor II la concentración necesaria para evitar la formación de precipitado debe ser superior a $50 \mathrm{ppm}$.

\section{Interacción Fluido - Fluido.}

Sludge. Al colocar en contacto los inhibidores I, II y III con el crudo del pozo CUP XL4, no se encontró formación de Sludge. La Figura 2 muestra las fotografías de la malla al finalizar la prueba.

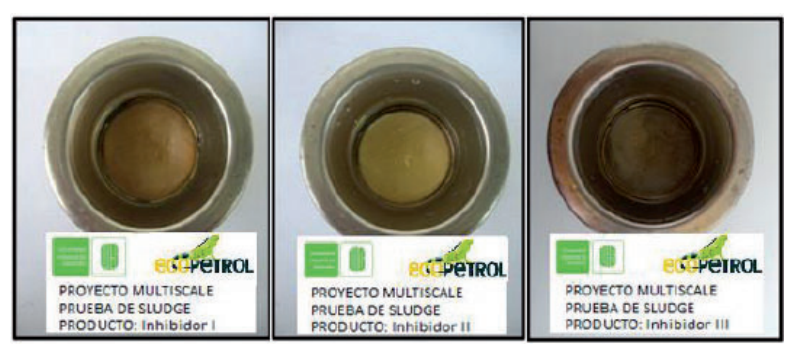

Figura 2. Prueba de Sludge (Inhibidores I, II y III)

Mojabilidad Visual. Los productos evaluados: Inhibidor I, II y III mostraron tendencia a humectar por agua la arena, lo cual indica que no cambian la mojabilidad del yacimiento.

Rompimiento de Emulsiones. Los resultados de las pruebas de rompimiento de emulsiones con los inhibidores I, II, III y el crudo del pozo CUP XL4, se encuentran en las Figuras 3, 4 y 5 en las cuales se muestra el porcentaje de rompimiento de cada uno de los inhibidores a las proporciones de $25-75,50-50$ y 75 25 respectivamente, durante los tiempos de 15 minutos (1), 1 hora (2) y 24 horas (3). 


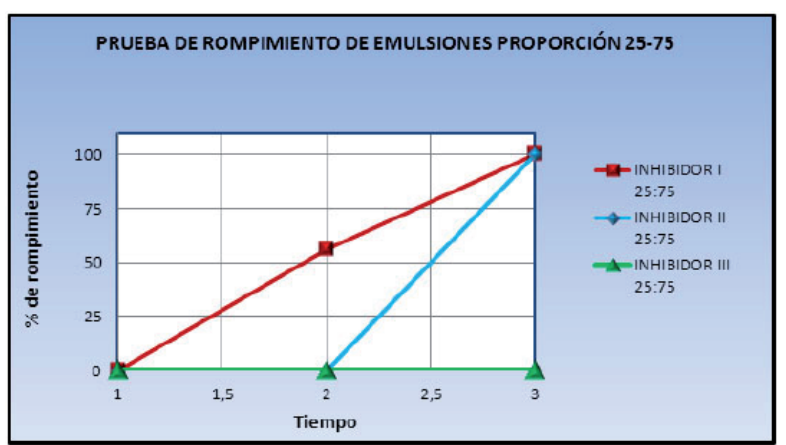

Figura 3. Rompimiento de emulsiones proporción 25-75 Inhibidor - Crudo CUP XL4

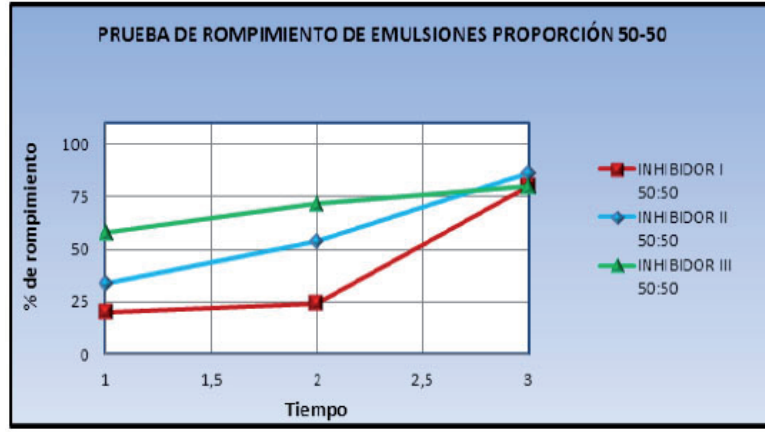

Figura 4. Rompimiento de emulsiones proporción 50-50 Inhibidor - Crudo CUP XL4

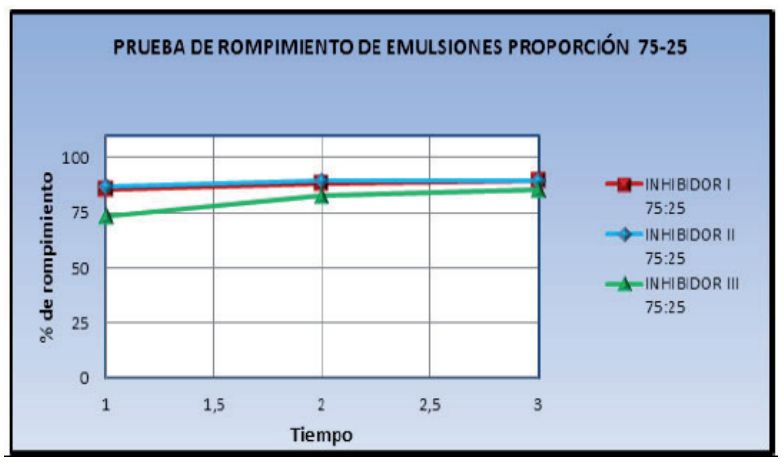

Figura 5. Rompimiento de las emulsiones, proporción 75-25 Inhibidor - Crudo CUP XL4.
Compatibilidad. En esta prueba se evaluó la presencia de sólidos en la fase acuosa y/o en la interfase, así como la apariencia de la mezcla. Con base en los resultados obtenidos se identificó posibles incompatibilidades. Los resultados de la prueba de compatibilidad con los fluidos del pozo CUP XL 4 y los inhibidores I, II, y III al finalizar las 24 horas de prueba se encuentran en la Tabla 4.

De acuerdo con los resultados obtenidos, los inhibidores I y III fueron compatibles con los fluidos del pozo CUP XL-4 sin envenenar, para todas las concentraciones evaluadas al término de 24 horas de observación y una temperatura de $95{ }^{\circ} \mathrm{C}$. El inhibidor II fue compatible para las concentraciones de $1 \%$ y $2,5 \%$ con agua y crudo, pero presentó incompatibilidad a las concentraciones de $5 \%, 10 \%$ y $20 \%$ con agua de formación sintética.

\section{Compatibilidad Envenenando con Calcio, Hierro y}

Bario. Los resultados de la prueba de compatibilidad envenenando los fluidos del pozo CUP XL4 con 5.000 ppm de Calcio, 5.000 ppm de Hierro y 500 ppm de Bario, al finalizar las 24 horas de prueba se encuentran en la Tabla 5.

De acuerdo con los resultados de la Tabla 5, el inhibidor I presentó el mejor comportamiento en las pruebas de compatibilidad con los fluidos del pozo CUP XL4 envenenados con Calcio, Hierro y Bario. El inhibidor III fue compatible en presencia de Hierro e incompatible en presencia de Calcio y Bario; y el inhibidor II presentó incompatibilidad en presencia de Calcio, Hierro y Bario.

La nomenclatura utilizada para presentar los resultados de las pruebas de compatibilidad se describe a continuación:

Compatible

Incompatible por precipitado

Tabla 4. Resultados de la prueba de compatibilidad con los fluidos del pozo CUP XL 4

\begin{tabular}{|c|c|c|c|c|c|c|c|c|c|c|}
\hline \multirow{3}{*}{ PRODUCTO } & \multicolumn{10}{|c|}{ CONCENTRACIONES } \\
\hline & \multicolumn{2}{|c|}{$1 \%$} & \multicolumn{2}{|c|}{$2,5 \%$} & \multicolumn{2}{|c|}{$5 \%$} & \multicolumn{2}{|c|}{$10 \%$} & \multicolumn{2}{|c|}{$20 \%$} \\
\hline & AGUA & CRUDO & AGUA & CRUDO & AGUA & CRUDO & AGUA & CRUDO & AGUA & CRUDO \\
\hline Inhibidor I & 0 & 0 & 0 & 0 & 0 & 0 & 0 & 0 & 0 & 0 \\
\hline Inhibidor III & O & O & O & 0 & $\bigcirc$ & 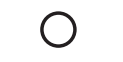 & 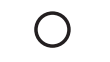 & O & O & 0 \\
\hline Inhibidor II & 0 & 0 & 0 & 0 & 0 & --- & 0 & --- & 0 & --- \\
\hline
\end{tabular}


Tabla 5. Resultados de la prueba de compatibilidad con los fluidos del pozo CUP XL4 envenenados con Calcio, Hierro y Bario.

\begin{tabular}{|c|c|c|c|c|c|c|}
\hline \multirow{3}{*}{ PRODUCTO } & \multicolumn{6}{|c|}{ CONCENTRACIONES } \\
\hline & \multicolumn{2}{|c|}{$\begin{array}{c}20(\%) \\
5.000(\mathrm{ppm}) \\
\text { de Calcio }\end{array}$} & \multicolumn{2}{|c|}{$\begin{array}{c}20(\%) \\
5.000(\mathrm{ppm}) \\
\text { de Hierro }\end{array}$} & \multicolumn{2}{|c|}{$\begin{array}{c}20(\%) \\
500(\text { ppm) } \\
\text { de Bario }\end{array}$} \\
\hline & AGUA & CRUDO & AGUA & CRUDO & AGUA & CRUDO \\
\hline Inhibidor I & & $\mathrm{O}$ & & & & 0 \\
\hline Inhibidor III & & --- & & 0 & & --- \\
\hline Inhibidor II & & --- & & --- & & \\
\hline
\end{tabular}

\section{Pruebas TÉcnicas.}

Estabilidad Térmica. En esta prueba se evaluó la estabilidad de los inhibidores y los posibles cambios en su estructura, al ser sometidos por 5 días a $122^{\circ} \mathrm{C}$ y 4.500 psi. De acuerdo con los resultados y los espectros de los productos se puede inferir que los inhibidores I y
II no sufren cambios en su estructura, ni se degradan al ser sometidos por 5 días a $122{ }^{\circ} \mathrm{C}$ y 4.500 Psi.

Eficiencia Dinámica. Los resultados de la prueba de eficiencia dinámica para los inhibidores I, II y III se resumen en la Tabla 6.

Tabla 6. Resultados de la Prueba de Eficiencia Dinámica.

\begin{tabular}{ccccccc} 
PRODUCTO & $\mathbf{5}$ & $\mathbf{6}$ & $\mathbf{7}$ & $\mathbf{1 0}$ & $\mathbf{2 0}$ \\
& $(\mathrm{ppm})$ & $(\mathrm{ppm})$ & $(\mathrm{ppm})$ & $(\mathrm{ppm})$ & $(\mathbf{p p m})$ \\
\hline Inhibidor I & - & - & $\bigcirc$ & $\bigcirc$ & $\bigcirc$ \\
\hline Inhibidor III & - & --- & - & $\bigcirc$ & $\bigcirc$ \\
\hline Inhibidor II & - & --- & --- & $\bigcirc$ & $\bigcirc$ \\
\hline
\end{tabular}

Los resultados reportados en la Tabla 6 están basados en la siguiente nomenclatura:

No ocurre depositación de incrustaciones

Tendencia incrustante

Depositación de incrustaciones

De acuerdo con los resultados mostrados en la Tabla 6, se pudo establecer la concentración mínima de inhibidor (MIC) a las condiciones de presión y temperatura de yacimiento. Para el Inhibidor I, la MIC fue de 7 ppm; para el inhibidor III fue de $10 \mathrm{ppm}$ y para el inhibidor II mayor de $10 \mathrm{y} \leq 20 \mathrm{ppm}$.

Interacción Fluido - Roca. Para determinar el valor de permeabilidad de referencia, después de la Restauración de Mojabilidad del Plug, se realizaron ciclos de inyección de crudo - salmuera hasta que los valores del diferencial de presión $(\Delta \mathrm{P})$, de los dos últimos ciclos variaran en menos del $5 \%$ durante $10 \mathrm{Vp}$ desplazados. Los resultados obtenidos se muestran en la Tabla 7.
Tabla 7. Comparación de los diferenciales de presión para los ciclos de crudo-salmuera.

\begin{tabular}{cccc} 
& FLUIDO & $\begin{array}{c}\mathbf{Q} \\
\left(\mathbf{c m}^{3} / \mathbf{m i n}\right)\end{array}$ & $\begin{array}{c}\overline{\Delta \mathbf{P}} \\
(\mathbf{P s i})\end{array}$ \\
\hline \multirow{2}{*}{ CICLO 1 } & Crudo & 1.0 & 75 \\
& Salmuera & 1.0 & 60 \\
CICLO 2 & Crudo & 1.0 & 99 \\
& Salmuera & 1.0 & 59 \\
CICLO 3 & Crudo & 1.0 & 65 \\
& Salmuera & 1.0 & 64 \\
CICLO 4 & Crudo & 1.0 & 60 \\
& Salmuera & 1.0 & 66 \\
CICLO 5 & Crudo & 1.0 & 91 \\
& Salmuera & 1.0 & 85 \\
CICLO 6 & Crudo & 1.0 & 94
\end{tabular}

En el ciclo 6 de crudo se obtuvo la condición esperada, ya que el $\Delta \mathrm{P}($ ciclo 6$)=\Delta \mathrm{P}($ ciclo 5$) \pm 5 \%$, por lo tanto se cumplió que las dos últimas permeabilidades al crudo fueron aproximadamente iguales con una diferencia de $5 \%$. Koil $=\operatorname{Koil}_{\mathrm{n}-1} \pm 5 \%$ 
El diferencial de presión de referencia correspondió al obtenido para el ciclo 6 de crudo. Posterior a esto se realizó la inyección de los preflujos orgánicos: PO1, PO2, PO3; Inhibidor I, $250 \mathrm{Vp}$ de salmuera, PO3, $\mathrm{PO} 2, \mathrm{PO} 1$ y finalmente se inyecto crudo en sentido de producción para obtener el diferencial de presión de retorno. En la Tabla 8 se realiza una comparación entre el diferencial de presión de referencia y de retorno.

Tabla 8. Comparación de los diferenciales de presión de referencia y retorno.

\begin{tabular}{cccc} 
& FLUIDO & $\begin{array}{c}\mathbf{Q} \\
\left(\mathbf{c m}^{3} / \mathbf{m i n}\right)\end{array}$ & $\begin{array}{c}\overline{\Delta \mathbf{P}} \\
(\mathbf{P s i})\end{array}$ \\
$\Delta \mathrm{P}$ de referencia & Crudo & 1.0 & 94 \\
\hline$\Delta \mathrm{P}$ de retorno & Crudo & 1.0 & 44.8 \\
\hline
\end{tabular}

Los resultados de la Tabla 8 muestran el efecto del inhibidor I sobre el diferencial de presión, comparando el $\Delta \mathrm{P}$ obtenido antes y después de la inyección del producto. Al observar el diferencial de presión se puede evidenciar una disminución en el valor de este que corresponde a 49.2 Psi. Con base en lo anterior se puede afirmar que el tratamiento realizado no ocasiona daño a la formación de interés y es compatible con el yacimiento. Debido a lo anterior, el inhibidor I se clasificó como apto para una inyección por squeeze en el pozo CUP XL4.

Igualmente se realizaron análisis fisicoquímicos a las soluciones acuosas recolectadas durante el desplazamiento, cuyos resultados se presentan en la Tabla 9.

Tabla 9. Análisis de fósforo en los efluentes del desplazamiento

\begin{tabular}{|c|c|c|c|c|}
\hline TUBOS & $\begin{array}{l}\text { VOLUMEN POROSO } \\
\left(\mathrm{cm}^{3}\right)\end{array}$ & $\begin{array}{l}\text { [INHIBIDOR] } \\
(\mathrm{mg} / \mathrm{L} \text { de } \mathrm{P})\end{array}$ & $\begin{array}{c}{[\text { INHIBIDOR] }} \\
\left(\mathrm{mg} / \mathrm{L} \mathrm{de} \mathrm{PO}_{4}{ }^{3-}\right)\end{array}$ & $\begin{array}{c}\text { [INHIBIDOR] } \\
\text { COMO PRODUCTO } \\
(\mathrm{mg} / \mathrm{L})\end{array}$ \\
\hline Inhibidor al 7,5 \% & - & 6971 & 21373,92 & 91733,57 \\
\hline Tubo 4 & 4 & 268,7 & 823,87 & 3535,91 \\
\hline Tubo 5 & 5 & 68,14 & 208,93 & 896,68 \\
\hline Tubo 6 & 6 & 53,27 & 163,33 & 701,00 \\
\hline Tubo 7 & 7 & 24,6 & 75,43 & 323,72 \\
\hline Tubo 8 & 8 & 15,7 & 48,14 & 206,60 \\
\hline Tubos 9-13 & 11 & 6,11 & 18,73 & 80,40 \\
\hline Tubos 14-23 & 18 & 2,29 & 7,02 & 30,13 \\
\hline Tubos 24-38 & 31 & 1,19 & 3,65 & 15,66 \\
\hline Tubos 39-58 & 48 & 0,741 & 2,27 & 9,75 \\
\hline Tubos 59-83 & 71 & 0,43 & 1,32 & 5,66 \\
\hline Tubos 84-103 & 93 & 0,34 & 1,04 & 4,47 \\
\hline
\end{tabular}

En la Figura 6 se puede observar la desorción del inhibidor I durante el desplazamiento de los 250 volúmenes porosos de salmuera.

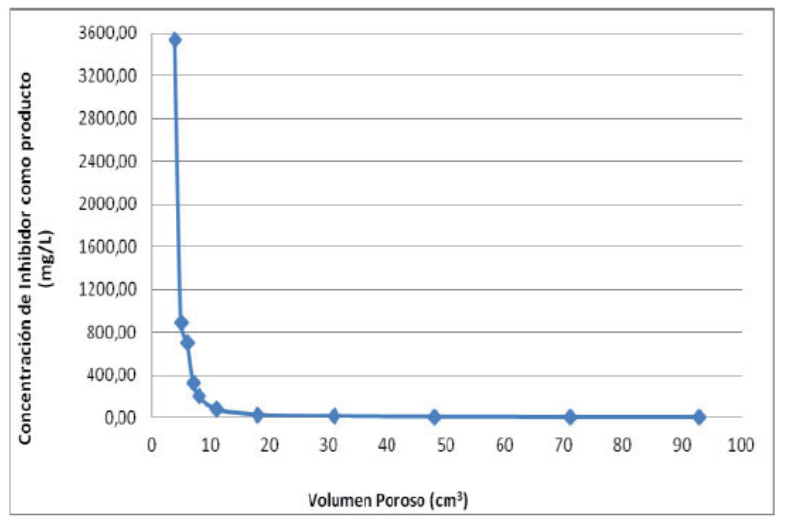

Figura 6. Desorción del inhibidor en los efluentes
Mediante la ecuación de la línea de tendencia de la Figura 6 se determinó que en el volumen poroso No 62 se obtenía una concentración de inhibidor igual a 7 ppm. De acuerdo con los resultados de la prueba de Eficiencia Dinámica por debajo de esta concentración el inhibidor no es eficiente para evitar la depositación de incrustaciones.

\section{CONCLUSIONES}

Los tres productos evaluados no presentaron Sludges al término de 24 horas y mostraron tendencia a humectar por agua la arena en la prueba de Mojabilidad.

De acuerdo con la comparación de los espectros de IR los inhibidores I y II fueron estables térmicamente a la temperatura y presión de fondo de pozo, mientras que el inhibidor III presentó degradación térmica al ser sometido durante 5 días a las mismas condiciones. 
Al evaluar la compatibilidad de los inhibidores con los fluidos y arena de la formación, solo el inhibidor I tuvo buenos resultados en todas las pruebas realizadas, mientras que el inhibidor II no tuvo buenos resultados en las pruebas de compatibilidad con y $\sin$ envenenamiento, y el inhibidor III no tuvo buenos resultados en las pruebas de rompimiento de emulsiones ni compatibilidad con envenenamiento.

Se determinó la concentración mínima de inhibidor para prevenir la formación de incrustaciones de $\mathrm{CaCO}_{3}$ y $\mathrm{BaSO}_{4}$ a condiciones dinámicas y de yacimiento. El inhibidor que tuvo la menor MIC a estas condiciones fue el inhibidor I con una $\mathrm{MIC}=7 \mathrm{ppm}$.

Al evaluar el inhibidor seleccionado (Inhibidor I) mediante pruebas de desplazamiento fluido-roca, se pudo establecer que el inhibidor es compatible con el yacimiento, por tanto no causa daño a la formación al ser inyectado en el pozo CUP XL-4.

Mediante el análisis de los efluentes se pudo establecer que la concentración mínima del inhibidor seleccionado se obtiene en el volumen poroso \# 62 de agua producida, posterior a la inyección del inhibidor. Esta información es primordial para determinar la durabilidad del tratamiento en el pozo evaluado, para lo cual se realiza un escalamiento.

\section{REFERENCIAS}

1. KELLAND, Malcolm A. "Effect of Various Cations on the Formation of Calcium Carbonate and Barium Sulfate Scale with and without Scale Inhibitors". En: I\&EC Research. Marzo, 2011. p. 5852-5861.

2. LEÓN, Orietta; CÁRDENAS, Carmen; CARRUYO, Jenny. "Costumed of scale inhibitors in oilfield water plant”. En: Rev. Téc. Ing. Unív. Zulia. 2002. Vol. 25, no. 1, p. 26-32.

3. JINES, José Luis. Uso del tubo de diálisis en el diseño de tratamiento antiescala en el pozo SA 110 del campo sacha en distrito amazónico ecuatoriano. Trabajo de grado previo a la obtención del Título de: Ingeniero en Petróleo. Escuela Superior Politécnica del litoral. Facultad de Ingeniería en Ciencias de la Tierra, 2010. 186 p.

4. PINZÓN, Carmen; GARCÍA, Lucas Santiago; RODRÍGUEZ, Diego Felipe. "Modelamiento Termodinámico para la Inhibición de Incrustaciones de Carbonato de Calcio en Sistemas Hidrocarburos".
En: Revista Ingeniería y Región. Vol. 6, no. 1, p. 3443.

5. CRABTREE, Mike, et al. "La lucha contra las incrustaciones - Remoción y prevención". Oilfield Review. Halliburton, 1999. p. 30-49.

6. CHAMPION TECHNOLOGIES. LINEAS DE PRODUCTOS. Especialidades Químicas para la Industria Petrolera, 2009, 32 p.

7. GPA ESTUDIOS Y SERVICIOS PETROLEROS S.R.L. Nota Técnica n ${ }^{\circ} 23,9 \mathrm{p}$.

8. CRISTÓBAL, Sandra. Estudio del tamaño de partícula en la precipitación de sales con implicaciones en la formación de "scales". Trabajo de grado para optar al título de Ingeniero Químico. Universidad Rey Juan Carlos. Escuela Superior de Ciencias Experimentales y Tecnología, 2009. 68 p.

9. PERTIERRA, Guillermo. Determinación del tamaño de partículas de $\mathrm{CaCO}_{3}$ y $\mathrm{CaSO}_{4}$ en presencia de inhibidores de formación de incrustaciones. Trabajo de grado para optar al título de Ingeniero Químico. Universidad Rey Juan Carlos. Escuela Superior de Ciencias Experimentales y Tecnología, 2010.67 p.

10. PONTÓN, Diego; PAMBABAY, Iliana. Análisis Técnico-Económico y Diseño de Estimulaciones Matriciales para incrementar la productividad del campo Sacha en Petroproducción. Trabajo de grado para optar al título de Ingeniero en Petróleo. Escuela Superior Politécnica del litoral. Facultad de Ingeniería en Ciencias de la Tierra, 2008, 274 p.

11. CHEN, T.; NEVILLE, A.; YUAN, M. "Efecto de la combinación de los inhibidores DETPMP y PPCA sobre la formación de incrustaciones de $\mathrm{CaCO}_{3}$ ". En: SPE. Mayo 2004, p. 1-7.

12. BARBA, Diana Lucia; MARTÍNEZ, Paola Elizabeth. Estudio y diseño de un sistema cerrado de reinyección de agua de formación para la Estación Central del Campo Sacha, Trabajo de grado para optar al título de Ingeniera en Petróleo. Escuela Politécnica Nacional. Facultad de Ingeniería en Geología y Petróleos, 2009. 172 p.

13. LOPEZ, Jefferson; MURILLO, John Peter. Dimensionamiento de un Sistema de Tratamiento de Agua de Formación Previo a su Disposición para un Campo en el Oriente Ecuatoriano. Trabajo de 
grado para optar al título de Ingeniero en Petróleo, Escuela Superior Politécnica del Litoral. Facultad de Ingeniería en Ciencias de la Tierra, 2002. 189 p.

14. LOPEZ, Ivan; TORRES, Fredy Enrique. Inhibición de los precipitados de Carbonato de Calcio en la cara de la Formación y sus alrededores. Trabajo de grado para optar al título de Ingeniero de Petróleos. Universidad Industrial de Santander. Facultad de Ingenierías Fisico-químicas. Escuela de Ingeniería de Petróleos, 1999. 240 p.

15. ROBUSTILLO, Ana Rosa. Estudio de la solubilidad de carbonatos y sulfatos con implicaciones en la formación de "scales". Trabajo de grado para optar al título de Ingeniero Químico. Universidad Rey Juan Carlos. Escuela Superior de Ciencias Experimentales y Tecnología, 2009. 67 p.

16. HE, Shiliang; KAN, Amy; TOMSON, Mason. "Mathematical Inhibitor Model for Barium Sulfate Scale Control, Department of Environmental Science and Engineering". En: Langmuir. 1996. Vol. 12, no. 7, p. 1901-1905.

17. CONTRERAS, Enrique. "Desarrollo y aplicación de la capacidad para realizar pruebas de desplazamiento dinámico en muestras de núcleos de perforación de pozos petroleros". En: Boletin IEE. Julio-Agosto 2001. p. 152-165.

Recepción: 21 de septiembre de 2016

Aceptación: 5 de noviembre de 2016 